\title{
Oficina de ginástica da Universidade Aberta à Terceira Idade: Relatos e vivências
}

\author{
Gymmastics workshop of the University Open to the Elderly: Reports and experiences \\ Taller de gimnasia de la Universidad Abierta a Mayores: Informes y experiencias
}

Recebido: 07/05/2021 | Revisado: 12/05/2021 | Aceito: 13/05/2021 | Publicado: 21/05/2021

Eliane da Assunção Conceição
ORCID: https://orcid.org/0000-0003-4504-5424
Universidade do Estado da Bahia, Brasil
E-mail: aelianeassuncao@ gmail.com
Luanda Souza Alves
ORCID: https://orcid.org/0000-0002-1613-5036
Universidade do Estado da Bahia, Brasil
E-mail: contato.luandatube@ gmail.com
Mayume Alves Santos
ORCID: https://orcid.org/0000-0003-3272-3580
Universidade do Estado da Bahia, Brasil
E-mail: mhayumy17@gmail.com
Brandel José Pacheco Lopes Filho
ORCID: https://orcid.org/0000-0001-6666-9637
Universidade do Estado de Minas Gerais, Brasil
E-mail: brandelfilho@gmail.com
Bruno de Freitas Camilo
ORCID: https://orcid.org/0000-0002-3039-2194
Universidade do Estado de Minas Gerais, Brasil
E-mail: brunodefreitascamilo@ yahoo.com.br

\section{Resumo}

Este estudo tem como objetivo relatar a experiência oriunda do projeto de extensão "Oficina de Ginástica da Universidade Aberta à Terceira Idade", conduzido por discentes do curso de Licenciatura em Educação Física da Universidade do Estado da Bahia, Campus X. Trata-se de estudo descritivo qualitativo, realizado por meio da análise de conteúdo. A presente investigação foi realizada entre março e dezembro de 2018. As aulas do projeto foram subdivididas em três momentos, incluindo atividade inicial, atividade principal e "volta à calma". Trinta idosas participaram das atividades da oficina de ginástica, as quais relataram que sua participação no projeto de extensão contribuiu para a melhora na qualidade de vida em aspectos relacionados à saúde física, mental, social e afetiva. Foi possível constatar o contentamento das idosas ao redescobrir o prazer ao realizar tarefas que envolviam danças, brincadeiras, diálogos e reflexões. Observou-se, ainda, que é necessário sensibilizar os homens idosos acerca da importância da prática de atividade física para a melhoria da saúde. A experiência obtida pelas monitoras possibilitou maior proximidade entre docente e discentes, fortalecendo os laços existentes, culminando na construção da presente pesquisa. A produção do conhecimento em questão reforça o papel da Universidade Pública de propiciar aos seus educandos atividades de ensino, pesquisa e extensão, de forma articulada, a fim de contribuir para a formação de profissionais autônomos e capazes de intervir positivamente na sociedade à qual estão inseridos.

Palavras-chave: Monitoria de extensão; Universidade; Exercício físico; Idoso; Envelhecimento.

\begin{abstract}
This study aims to report the experience arising from the extension project "Gymnastics Workshop of the University Open to the Elderly", conducted by students of the Physical Education Degree course at the State University of Bahia, Campus X. This is a qualitative descriptive study, carried out through content analysis method. The present investigation was carried out between March and December 2018. The classes of the project were subdivided into three moments, including warm up and stretching activities, main activities and cool down phase. Thirty elderly women participated in the gymnastics workshop activities, reporting that their participation in the extension project contributed to the improvement in quality of life in aspects related to physical, mental, social and emotional health. It was possible to verify the contentment of the participants when they rediscovered the pleasure of performing tasks that involved dances, games, dialogues and reflections. It was also observed that it is necessary to raise awareness among older men about the importance of physical activity for improving health. The experience obtained by the student monitors enabled greater proximity between teacher and students, strengthening the existing bonds, culminating in the present research. This intellectual colaboration reinforces the role of the Brazilian Public University to provide its students with teaching, research and extension activities in an articulated way, contributing to the formation of autonomous professionals who are able to intervene in the society.
\end{abstract}

Keywords: Extension monitoring; Universities; Exercise; Aged; Aging. 


\begin{abstract}
Resumen
Este estudio tiene como objetivo reportar la experiencia derivada del proyecto de extensión "Taller de Gimnasia de la Universidad Abierta a Mayores", realizado por estudiantes de la Licenciatura en Educación Física de la Universidad Estadual de Bahía, Campus X. Se trata de un estudio cualitativo descriptivo, realizado mediante análisis de contenido. Esta investigación se llevó a cabo entre marzo y diciembre de 2018. Las clases del proyecto se subdividieron en tres momentos, incluida la actividad inicial, la actividad principal y "volver a la calma". Treinta ancianas participaron de las actividades del taller de gimnasia, las cuales informaron que su participación en el proyecto de extensión contribuyó a la mejora de la calidad de vida en aspectos relacionados con la salud física, mental, social y emocional. Se pudo constatar la alegría de las ancianas cuando redescubrieron el placer de realizar tareas que involucraban bailes, juegos, diálogos y reflexiones. También se observó que es necesario concientizar a los hombres mayores sobre la importancia de la actividad física para mejorar la salud. La experiencia obtenida por los monitores permitió una mayor proximidad entre profesores y alumnos, fortaleciendo los lazos existentes, culminando con la construcción de esta investigación. Esta producción de conocimiento refuerza el papel de la Universidad Pública Brasileña en brindar actividades articuladas de docencia, investigación y extensión a sus estudiantes, contribuyendo a la formación de profesionales autónomos capaces de intervenir positivamente en la sociedad.
\end{abstract}

Palabras clave: Monitoreo de extensiones; Universidad; Ejercicio físico; Anciano; Envejecimiento.

\title{
1. Considerações Iniciais
}

O crescimento populacional de indivíduos com idade igual ou superior a 60 anos tem sido observado em todo o mundo (United Nations, 2020). No Brasil, as alterações no perfil etário ocorreram de forma acelerada, como no restante do mundo, impactando em grandes desafios para a compreensão do envelhecimento e de suas implicações para a sociedade (Borges, Campos, Castro \& Silva, 2015).

O processo de envelhecimento está associado a modificações na vida dos indivíduos a partir das esferas física, cognitiva, social, dentre outras, podendo influenciar em sua autonomia e independência (World Health Organization, 2017). Estas alterações podem ser naturais, quando ligadas a alterações biológicas e fisiológicas, em decorrência da senescência, ou patológicas, quando agravadas por quadros clínicos diversos (Almeida \& Reis, 2016) que demandam cuidados diferenciados (Cunha, Cunha \& Barbosa, 2016).

As mudanças provenientes do envelhecimento humano, tais como diminuição de massa óssea, danos às estruturas cartilaginosas, redução da elasticidade dos ligamentos, perda de força muscular e infiltração gordurosa nos tecidos podem contribuir para o desenvolvimento de doenças como osteoporose, sarcopenia, osteoartrite e artrite inflamatória (Melo, Nakatani, Pereira, Menezes \& Pagotto, 2017). Além disso, a manutenção de um estilo de vida pouco ativo, com baixos níveis de atividade física e elevado tempo em comportamento sedentário, pode comprometer a saúde, ao longo dos anos (Camilo, 2019).

A partir desse contexto, o projeto de extensão "Oficina de Ginástica da Universidade Aberta à Terceira Idade" (UATI) foi criado em 2017, com o intuito de realizar a promoção da saúde e melhoria da qualidade de vida dos idosos por meio da prática regular de atividade física. A Extensão Universitária faz parte da matriz curricular da Educação Superior Brasileira, caracterizando-se como um processo interdisciplinar construtivo de cunho cultural, educacional, científico, político e tecnológico, que estabelece a comunicação entre as instituições de ensino superior (IES) e outras esferas da sociedade por meio da sua associação com o ensino e a pesquisa (Ministério da Educação, 2018). Compreende, em sua prática, atividades de monitoria acadêmica, as quais visam intensificar e garantir a cooperação entre discente e docente nas tarefas essenciais da Universidade, no que se refere ao ensino, à pesquisa e à extensão. A monitoria de extensão, portanto, é oferecida aos discentes regularmente matriculados no curso de graduação da IES, concebendo atribuições colaborativas, equivalentes às atividades acadêmicas, e configurando-se como uma possibilidade de aprendizado construído por meio da teoria articulada às práxis pedagógicas (Conselho Universitário, 2016).

Acredita-se que a participação de graduandos em projetos de extensão permite o aprofundamento de conhecimentos específicos da área e sua inter-relação com diversos saberes. Além disso, contribui para a aproximação entre docente e 
graduandos (Nozaki, Ferreira \& Hunger, 2015), tornando o processo de ensino-aprendizagem mais cooperativo e viabilizando a produção de conhecimento dos discentes envolvidos no projeto de extensão.

Evidências da literatura demonstram que a prática de atividade física apresenta efeitos benéficos para a saúde e qualidade de vida das pessoas com idade mais avançada (Lima \& Camilo, 2019; Madeira \& Pacheco, 2011; Paiva, Fagundes, Amaral \& Pacheco, 2014). A partir de um estudo de revisão, evidenciou-se que a dança praticada por idosos, independentemente do estilo, contribui para a melhoria da qualidade de vida nos aspectos físicos, psicológicos, fisiológicos, relações sociais e meio ambiente (Lima \& Camilo, 2019). Além disso, na pesquisa desenvolvida em um Núcleo de Apoio à Saúde da Família (NASF) no município de Catingueira, Paraíba, constatou-se que as idosas participantes do projeto FELIZ idade relataram maior empolgação (Camboim et al., 2017). Outrossim, o relato de experiência realizado por Silva, Messias, Paixão, Sena \& Silva (2019) constatou que a prática de ginástica localizada realizada por idosos no projeto de extensão universitário multidisciplinar "Universidade Aberta à Terceira Idade" da Universidade Estadual de Ciências da Saúde de Alagoas contribuiu para a melhoria de aspectos físicos e, também, relacionados à socialização. Nota-se, portanto, que projetos de extensão desenvolvidos em IES podem estimular a promoção de um estilo de vida mais ativo e saudável em pessoas com idade avançada, a partir da prática regular de atividade física.

Assim, este estudo tem como objetivo relatar a experiência oriunda do projeto de extensão "Oficina de Ginástica da Universidade Aberta à Terceira Idade", conduzido por discentes do curso de licenciatura em Educação Física da Universidade do Estado da Bahia (UNEB), Campus X. De forma secundária, objetivou-se descrever as atividades realizadas, bem como a participação das discentes e do docente, coordenador do projeto de extensão.

\section{Métodos}

\subsection{Caracterização do estudo}

Este relato é caracterizado como descritivo qualitativo, seguindo os princípios da análise de conteúdo (BARDIN, 2000; VALA, 2003). A abordagem qualitativa é um campo multifacetado, marcado por diferentes orientações e métodos, que permite realizar uma investigação aprofundada de uma variedade de temas relacionados à realidade singular ou às múltiplas realidades, capturando o significado de fenômenos subjetivos na perspectiva dos participantes do estudo (Yin, 2016).

A presente pesquisa foi desenvolvida a partir do projeto de extensão da "Oficina de Ginástica da Universidade Aberta à Terceira Idade", realizado na Universidade do Estado da Bahia (UNEB), Campus X, Teixeira de Freitas - BA, entre março e dezembro de 2018. O projeto de extensão foi amplamente divulgado na comunidade e poderiam se inscrever pessoas de ambos os sexos, com idade $\geq 60$ anos que apresentassem um atestado médico informando sua aptidão para realizar atividades físicas. Foram reunidos os depoimentos escritos das participantes do projeto, os quais serão discutidos ao longo do texto. A coleta dos depoimentos foi realizada ao final das aulas, a partir da seguinte pergunta norteadora: "De que forma a prática das atividades desenvolvidas na Oficina de Ginástica contribuiu para melhoria da sua qualidade de vida?" Para manter as identidades das depoentes em sigilo e permitir maior liberdade de expressão, os relatos não possuíram nenhuma forma de identificação, sendo nomeados aqui por espécies de pássaros, escolhidas arbitrariamente. A escolha das participantes se deu por conveniência, através da participação voluntária, e todas concederam autorização para uso de seus escritos.

\subsection{Descrição das atividades do projeto de extensão}

As atividades foram desenvolvidas semanalmente, com encontros às segundas e quartas-feiras, com duração de 60 minutos em cada aula, as quais foram conduzidas por quatro graduandas do curso de licenciatura em Educação Física da UNEB, Campus X, sob a supervisão do professor coordenador do projeto de extensão. Para o desenvolvimento das aulas, utilizou-se os seguintes recursos materiais: aparelho de som, cadeiras, bolas de borracha, arcos, bastões, colchonetes, caneleiras, halteres, papel e caneta. A dinâmica dos encontros possuía três momentos, que incluíam a atividade inicial, a 
atividade principal e a "volta à calma". Na atividade inicial realizavam-se alongamentos, a fim de preparar as participantes para a rotina do dia. A atividade principal era constituída de treinamento de força, dança e movimentos de luta ou circuito, de acordo com programação estabelecida previamente pelas monitoras e coordenador do projeto. A "volta à calma" era desenvolvida por meio de exercícios de relaxamento que estimulavam o controle da respiração e a redução da frequência cardíaca.

A cada semana uma monitora era responsável por conduzir as atividades, com auxílio das demais monitoras e sob a supervisão do coordenador. Os planos de aula a serem desenvolvidos, ao longo do mês, eram enviados com antecedência para que o professor realizasse as devidas considerações antes de sua execução. As aulas apresentavam objetivos distintos e foram desenvolvidas a partir de atividades de estímulo cognitivo, alongamentos, exercícios para o aumento/manutenção da força, coordenação motora e equilíbrio.

As atividades foram organizadas em: (1) treinamento de força; (2) abdominais; (3) dança; (4) movimentos básicos de lutas; (5) ginástica aeróbica, com movimentos básicos ao som de músicas; e (6) exercícios de flexibilidade, coordenação e equilíbrio. O Quadro 1 reúne os exercícios desenvolvidos nas aulas.

Quadro 1. Exercícios realizados nas aulas do projeto de extensão.

\begin{tabular}{|l|l|l|}
\hline \multicolumn{1}{|c|}{ Tipo } & \multicolumn{2}{c|}{ Exercícios } \\
\hline \multirow{2}{*}{ Treino de Força } & $\begin{array}{l}\text { Membros } \\
\text { Superiores }\end{array}$ & $\begin{array}{l}\text { Flexão, abdução e desenvolvimento de ombros; rosca simultânea e } \\
\text { alternada; tríceps coice e francês; remada curvada; crucifixo invertido; } \\
\text { flexão de braço com apoio de joelhos; supino e crucifixo com halteres }\end{array}$ \\
\cline { 2 - 3 } & $\begin{array}{l}\text { Membros } \\
\text { Inferiores }\end{array}$ & $\begin{array}{l}\text { Agachamento livre e sumô; flexão de quadril (em pé e no solo); } \\
\text { abdução de quadril (em pé e no solo), adução de quadril (solo); flexão } \\
\text { de joelhos (em pé e no solo); extensão de quadril (em pé e no solo); } \\
\text { panturrilha em pé (com apoio na parede) }\end{array}$ \\
\hline Abdominais & $\begin{array}{l}\text { Abdominal reto 90; abdominal remador; abdominal oblíquo (em pé e no solo); } \\
\text { prancha isométrica }\end{array}$ \\
\hline Dança & $\begin{array}{l}\text { Exercícios com foco em percepção de espaço, desenvolvimento motor e movimento } \\
\text { básicos dos membros superiores e inferiores ao som de diferentes estilos musicais. }\end{array}$ \\
\hline Lutas & Exercícios de Muay Thai: jab, direto, cruzado, joelhada e upper \\
\hline Ginástica Aeróbica & \multicolumn{2}{|l|}{ Movimentos articulares de flexão, extensão, abdução e adução } \\
\hline Flexibilidade & \multicolumn{2}{|l|}{ Alongamentos balísticos } \\
\hline
\end{tabular}

Fonte: Autores.

Também foram realizados circuitos envolvendo atividades de coordenação motora, lateralidade e movimento de marcha, organizados em estações. Na primeira estação foram colocados arcos no chão, para que as idosas pisassem dentro, com o intuito de desenvolver a lateralidade (direita e esquerda). A segunda estação foi constituída de colchonetes para a realização de abdominais ou de outros exercícios com caneleira ou halteres. Na terceira estação, foram utilizados mini cones ou garrafas plásticas com areia, para o desenvolvimento da marcha. Na quarta estação, cama elástica, bastão e corda foram utilizados a fim de estimular a manutenção/melhora da coordenação motora, agilidade e equilíbrio. As atividades desenvolvidas em cada estação possuíam duração de dois minutos, com intervalo de um minuto entre as estações.

$\mathrm{O}$ treinamento de força foi realizado a partir de exercícios de membros superiores e inferiores, com método alternado por seguimento, cujo intuito foi evitar a fadiga muscular precoce. Os exercícios foram realizados a partir de duas a três séries, com variação entre oito e 15 repetições em cada exercício. O intervalo de descanso entre as séries variou entre 30 e 60 segundos e a progressão de carga foi realizada gradualmente, conforme as idosas relatavam facilidade para realizar o 
movimento. Ao final de algumas aulas disponibilizava-se alguns minutos para diálogo acerca de assuntos diversos relacionados ao cotidiano das participantes. Esse momento de diálogo possibilitava a partilha de experiências individuais e coletivas, fornecendo informações, às monitoras e ao coordenador, para aprimorar o planejamento das atividades a serem desenvolvidas nas aulas.

\section{Resultados e Discussão}

Trinta idosas $(\mathrm{n}=30)$ participaram do projeto de extensão "Oficina de Ginástica da Universidade Aberta à Terceira Idade" (UATI/CEVIT) durante o período de março a dezembro de 2018. As idosas possuíam idade entre 60 e 82 anos e se autodeclararam casadas, solteiras ou viúvas. As participantes eram aposentadas ou pensionistas e possuíam grau de escolaridade que variou entre o ensino fundamental e médio.

As idosas relataram grande interesse e motivação para a prática regular de atividade física. Além disso, destacaram que a participação nas atividades do projeto de extensão proporcionou melhora na saúde física, mental, social e afetiva. A seguir, são apresentados alguns trechos de relatos feitos acerca da influência dessas atividades sobre a saúde:

Ajudou a fortalecer os ossos para andar e disposição para fazer as coisas. (Azulão)

Teve muita melhoria. Melhorou as minhas dores nas pernas, minhas costas e também passei a ter disposição para fazer as coisas. (Andorinha)

Pra [sic] mim é muito bom. Quando chego aqui sinto alegria e disposição. Diminuiu muito minhas dores das pernas, coluna e pescoço. (Curió)

Apesar do envelhecimento ser acompanhado de mudanças fisiológicas e comportamentais que podem ocasionar problemas à saúde (Camilo, 2021), evidências da literatura denotam os efeitos positivos da atividade física para pessoas com idade mais avançada (Lima \& Camilo, 2019; Moreno \& Tsukamoto, 2018). Nesta linha, o relato de experiência de Garcia, Vale, Ribeiro \& Biagini (2020), envolvendo discentes que participaram de um projeto de extensão acerca do treinamento funcional, evidenciou que os idosos que praticavam atividade física orientada relataram melhora na qualidade de vida, autoestima e na confiança ao realizar atividades da vida diária. Além disso, um estudo de revisão demonstrou que a prática de atividade física reduziu as dores musculares e ósseas, possibilitando aos idosos maior mobilidade e autonomia para realizar suas atividades cotidianas (Menezes, Silva, Silvério \& Medeiros, 2020). Tais dados estão em consonância com a presente investigação, reforçando a necessidade de se manter fisicamente ativo, a fim de atenuar os efeitos deletérios advindos do avançar da idade.

No que se refere à saúde mental, as idosas participantes do projeto relataram que as atividades proporcionaram redução da ansiedade e dos sintomas depressivos. Os trechos abaixo reúnem estes depoimentos:

Tirou ansiedade, tirou minha vontade de ficar somente em casa. Melhorou minha musculatura, desenvolvimento mental, porque estava parada, e também a depressão. As monitoras são maravilhosas. CEVIT foi uma grande descoberta. (Lavadeira)

Contribuiu muito. Melhorou mais a minha depressão, diminuiu as dores musculares. Nota mil. As meninas [são] uns amores. Eu amo CEVIT. (Jandaia)

Melhorou minha saúde, meu estado emocional. (Anu) 
Conforme evidenciado, as participantes relataram melhoria em diversos aspectos relacionados à qualidade de vida, especialmente quanto a fatores emocionais e de bem-estar psicológico, como sintomas de ansiedade e depressão. Pessoas com idade mais avançada podem apresentar ansiedade, insegurança, baixa autoestima, dependência, depressão e solidão, a depender do seu contexto de vida. Apesar disso, a prática regular de atividade física pode ser um adjuvante neste processo, contribuindo para a redução de problemas de saúde relacionados aos transtornos mentais e, ainda, impactando positivamente sobre os aspectos emocionais (Araújo, Tolosa \& Zanella, 2015; Batista, Telles \& Sarraceni, 2016) e psicológicos (Araújo et al., 2015; Batista et al., 2016; Lima \& Camilo, 2019).

A partir de um estudo envolvendo 24 revisões sistemáticas e metanálises, constatou-se que idosos que apresentavam maiores níveis de atividade física possuíam menor chance de desenvolver depressão, quando comparados àqueles que não realizaram, ao menos, 150 minutos de atividade física por semana (Cunningham, Sullivan, Caserotti \& Tully, 2020). Além disso, Blay, Andreoli, Fillenbaum \& Gastal (2007) demonstraram a existência de uma relação direta e positiva entre a prática de atividade física e a sintomatologia depressiva. Os autores (ibidem) sugerem que a atividade física exerce um efeito protetor sobre os sintomas depressivos, em conformidade com Batista et al. (2016), que afirma que tais atividades promovem um novo significado existencial, motivação autodeterminada e melhora da interação social. Em outro estudo, realizado a partir de um relato de experiência feito por discentes dos cursos de Educação Física e Fisioterapia, constatou-se que os idosos que participaram do programa de atividade física ofertado no Laboratório de Exercício Resistido em Saúde, da Universidade do Estado do Pará, apresentaram melhoria do bem-estar físico, emocional e psíquico (Queiroz et al., 2020), reforçando os achados da presente investigação.

Dentre os aspectos observados a partir da participação da oficina de ginástica, as idosas também relataram efeitos positivos na qualidade do sono e na socialização, conforme é destacado nos relatos a seguir:

Melhoria na qualidade do sono, disposição para realizar as atividades domésticas, redução de dores musculares, aumento da autoestima e diminuição da tristeza. (Bem-te-vi.)

Melhorou tudo. Quando não venho eu sinto falta. (Bacurau)

Alterações no padrão de sono devido a doenças físicas, comprometimentos mentais ou por modificações do ciclo circadiano são comuns, em idosos, e podem impactar negativamente na saúde e qualidade de vida (Haimov \& Shatil, 2013). A partir de um estudo que analisou a satisfação com o sono na percepção da qualidade de vida de idosos, foi possível constatar que o meio ambiente, as oportunidades de lazer e as relações sociais correlacionaram-se positivamente com o sono (Nascimento, Sonati, Martino \& Rodrigues, 2020). Deste modo, além da melhoria na qualidade do sono, a realização de atividades físicas fora do contexto domiciliar oportuniza aos idosos o aumento da interação social em diferentes espaços, tornando essa prática mais atrativa e motivadora. Somado a isso, a atividade realizada de forma coletiva, de forma semelhante ao que ocorre em projetos de extensão, pode contribuir para o estreitamento de vínculos entre seus pares. Ademais, as atividades desenvolvidas no projeto de extensão podem ser encaradas como uma oportunidade de lazer, uma vez que o idoso poderá vivenciar experiências prazerosas que não constituem atividades discricionárias.

Ao final de cada aula eram disponibilizados alguns minutos para um diálogo aberto, o qual era permeado por emoções e histórias de vida, induzindo assim uma troca mútua de experiências e ensinamentos, além de momentos de descontração e atividades relaxantes, como realização de massagens. Esses momentos possibilitaram aos envolvidos (idosas, monitoras e coordenador) o estreitamento de vínculos que pode ter contribuído para o aumento da autoestima e a valorização pessoal. Além disso, as participantes relatavam como se sentiam após a realização das atividades propostas, colaborando de forma ativa no 
planejamento das ações desenvolvidas no projeto.

$\mathrm{O}$ avançar da idade demarca um ponto importante do processo de envelhecimento, no qual o idoso tende a apresentar uma diminuição da sua capacidade para o trabalho, que pode culminar na aposentadoria. De acordo com Herdy (2020), o envelhecimento e a aposentadoria caminham paralelamente; entretanto, são fases da vida que podem ocorrer de forma independente. Tanto o envelhecimento quanto a aposentadoria podem contribuir para a redução das relações sociais externas, relacionadas ao trabalho, levando os idosos a permanecerem mais tempo em casa (Camilo, Meneguci, Tribess, Virtuoso Júnior \& Damião, 2019). Assim, projetos de extensão como a "Oficina de Ginástica da Universidade Aberta à Terceira Idade" podem incentivar os idosos a se tornarem fisicamente mais ativos, além de contribuir para a melhora da socialização, construção de vínculos afetivos e aumento da sensação de pertencimento ao meio em que está inserido.

\section{Considerações Finais}

Percebe-se a necessidade de refletir acerca da influência da atividade física sobre a saúde do idoso, considerando-se, principalmente, o crescimento populacional desse grupo etário específico. O objetivo do projeto de extensão aqui relatado foi proporcionar às idosas participantes mais disposição, bem-estar, melhoria da qualidade de vida, autonomia e maior socialização.

As idosas relataram que a participação no projeto foi benéfica para a saúde, contribuindo para a melhoria de aspectos físicos, sociais, emocionais e psicológicos. É importante salientar que a soma de todos estes benefícios resulta no cumprimento de objetivos mais amplos relacionados ao projeto de extensão, dentre eles, a melhora da qualidade de vida. Apesar dos relatos evidenciarem mudanças positivas percebidas pelas participantes, a ausência de indivíduos do sexo masculino indica uma necessidade de sensibilizar homens idosos acerca da importância da prática regular de atividades físicas em seu cotidiano, como estratégia de autocuidado, visando a melhora da saúde e o aumento da autoestima.

Destaca-se que todas as idosas participaram ativamente das práticas do projeto, mesmo considerando limitações físicas e especificidades de cada participante. Também foi possível observar o contentamento delas ao redescobrir o prazer durante a realização de tarefas que envolviam atividades de locomoção, danças, brincadeiras, diálogos e reflexões. Ao final de cada aula ocorria um momento de diálogo aberto, repleto de emoções, histórias de vida e troca mútua de experiências, que contribuiu de forma ímpar com o desenvolvimento do projeto. Os vínculos construídos entre as idosas, monitoras e coordenador em todo o percurso da Oficina de Ginástica foram essenciais, uma vez que promoveram mudanças significativamente positivas na vida de todos os envolvidos neste processo.

Participar da monitoria do programa da UATI tornou-se uma oportunidade ímpar para o desenvolvimento das discentes do curso de Licenciatura em Educação Física. A experiência obtida pelas graduandas, monitoras deste projeto, possibilitou maior proximidade entre docente e discentes, fortalecendo os laços existentes. Além disso, essa vivência possibilitou a construção da presente pesquisa, contribuindo com a produção do conhecimento e reforçando o papel da Universidade Pública de propiciar aos seus educandos atividades de ensino, pesquisa e extensão, de forma indissociável. Ademais, o desenvolvimento de projetos desta natureza pode contribuir para a formação de profissionais mais autônomos e capazes de intervir positivamente na sociedade à qual estão inseridos. Espera-se que estes dados contribuam com a discussão do tema, reunindo um maior número de subsídios para a formação de novas iniciativas e projetos universitários.

\section{Referências}

Almeida, R.F.F. \& Reis, A.O.A. (2016). Análise da produção científica no Brasil sobre envelhecimento e quedas. Revista Brasileira de Ciências do Envelhecimento Humano, 2(13), 242-253.

Araújo, G.H.C., Tolosa, L.B. \& Zanella, A.L. (2015). Benefícios da musculação sobre a qualidade de vida na terceira idade. Lecturas: Educación Física y Deportes, 20(205), 1. 
Bardin, L. (2000). Análise de Conteúdo. Lisboa: Edições 70.

Batista, A.M., Telles, J.D. \& Sarraceni, J.M. (2016). Efeitos do exercício físico sobre a qualidade de vida de idosos do projeto melhor idade: estudo comparativo. Revista Científica do Unisalesiano, 7(15), 399-411.

Blay, S., Andreoli, S., Fillenbaum, G. \& Gastal, F.L. (2007). Depression morbidity in later life: prevalence and correlates in a developing country. The American Journal of Geriatric Psychiatry, 15(9), 790-799.

Borges, G.M., Campos, M.B., Castro e Silva, L.G. (2015) Transição da estrutura etária no Brasil: oportunidades e desafios para a sociedade nas próximas décadas. In Mudança demográfica no Brasil no início do século XXI: subsídios para as projeções da população (pp. 138-151). Rio de Janeiro: Instituto Brasileiro de Geografia e Estatística.

Camboim, F.E.F., Nóbrega, M.O., Davim, R.M.B., Camboim, J.C.A., Nunes, R.M.V. \& Oliveira, S.X. (2017). Benefícios da atividade física na terceira idade para a qualidade de vida. Revista de Enfermagem da UFPE on line, 11(6), 2415-2422.

Camilo, B. F. (2019). Sentar-se menos e mover-se mais: o papel da atividade física na prevenção da obesidade. Arquivos de Ciências do Esporte, 7(3), 100.

Camilo, B.F., Meneguci, J., Tribess, S., Virtuoso Júnior, J.S. \& Damião, R. (2019). Associação combinada e independente do comportamento sedentário e atividade física com sobrepeso e obesidade em idosos. Revista Iberoamericana de Psicología del Ejercicio y el Deporte, 15(1), 19-25.

Camilo, B. F. (2021). Atividade física, comportamento sedentário e excesso de peso em idosos: um estudo longitudinal. Tese de doutorado, Universidade Federal do Triângulo Mineiro, Uberaba. MG, Brasil. http://bdtd.uftm.edu.br/handle/tede/373?locale=en.

Conselho Universitário. (2016). Resolução CONSU no 1.196/2016: Aprova as alterações no Regulamento do Programa de Bolsas de Extensão (PROBEX). Estado da Bahia. https://portal.uneb.br/proex/wp-content/uploads/sites/62/2017/10/1196-consu-Res.-Aprova-Regulamento-PROBEX-2.pdf

Cunha, A. C. N. P., Cunha, N. N. P. \& Barbosa, M. T. (2016). Geriatric teaching in Brazilian medical schools in 2013 and considerations regarding adjustment to demographic and epidemiological transition. Revista da Associação Médica Brasileira, 2 (62), 179-183.

Cunningham, C., O’ Sullivan, R., Caserotti, P., \& Tully, M. A. (2020). Consequences of physical inactivity in older adults: A systematic review of reviews and meta-analyses. Scandinavian Journal of Medicine \& Science in Sports, 30(5), 816-827.

Garcia, M.C.C., Vale, M.M., Ribeiro, J.C.T. \& Biagini, A.P. (2020). Experiências vivenciadas no programa de extensão "Atividade física funcional e recreativa para terceira idade" por discentes do curso de fisioterapia. Em Extensão, 19(2), 125-136.

Haimov, I. \& Shatil, E. (2013). Cognitive Training Improves Sleep Quality and Cognitive Function among Older Adults with Insomnia. Plos One, 8(4), 1-17.

Herdy, J.S. (2020). Envelhecimento: aposentadoria e velhice - fases da vida. GIGAPP Estudios Working Papers, 7(152), 242-260.

Lima, N. R. \& Camilo, B. F. (2019). A influência da dança na qualidade de vida dos idosos: uma revisão sistemática. Arquivos de Ciências do Esporte, 7(3), $140-143$.

Madeira, R. B. \& Pacheco, L. F. (2011). Estudo comparativo entre treinamento resistido convencional e treinamento resistido funcional em idosos. Lecturas: Educación Física y Deportes, 16(159), 1.

Melo, A.C.F., Nakatani, A.Y.K., Pereira, L.V., Menezes, R.L. \& Pagotto, V. (2017). Prevalência de doenças musculoesqueléticas autorreferidas segundo variáveis demográficas e de saúde: estudo transversal de idosos de Goiânia/GO. Cadernos Saúde Coletiva, 25(2), 138-143.

Menezes, G.R.S., Silva, A.S., Silvério, L.C., \& Medeiros, A.C.T. (2020). Impacto da atividade física na qualidade de vida de idosos: Uma revisão integrativa. Brazilian Journal of Health Review, 3(2), 2490-2498.

Ministério da Educação. (2018). Resolução $\mathrm{n}^{\circ} \quad 7$, de 18 de dezembro de 2018. Ministério da Educação. http://portal.mec.gov.br/index.php?option=com_docman\&view=download\&alias=104251-rces007-18\&category_slug=dezembro-2018-pdf\&Itemid=30192.

Moreno, N. L. \& Tsukamoto, M.H.C. (2018). Influências da prática da Ginástica Para Todos para a saúde na velhice: percepções dos praticantes. Conexões: Educação Física, Esporte e Saúde, 16(4)468-487.

Nascimento, E.F.A., Sonati, J.G., Martino, M.M.F. \& Rodrigues, J. (2020). O sono no contexto da qualidade de vida de idosos. Revista Mineira de Enfermagem, 24(e-1285), 1-6.

Nozaki, J. M., Ferreira, L. A., \& Hunger, D. A. C. F. (2015). Evidências formativas da extensão universitária na docência em Educação Física. Revista Eletrônica de Educação, 9(1), 228-241.

Paiva, T.A., Fagundes, R.R., Amaral, L.E.F. \& Pacheco, L.F. (2014). Doença de Parkinson e Exercícios Físicos: Possíveis benefícios. Revista Movimenta, $7(2), 700-710$.

Queiroz, G.V.R., Queiroz, T.Y.F.R., Souza, N.O., Nogueira, M.M., Santo, R.L.M., Silva, Y.G., Pereira, F.G. \& Souza, C.S.P. (2020). A relevância das atividades físicas como instrumento para saúde mental dos idosos: um relato de experiência/The relevance of physical activities as an instrument for mental health of the elderly: an experience report. Brazilian Journal of Health Review, 3(6), 16944-16950.

Silva, M.A.M., Messias, J.M.O., Paixão, J.T.S., Sena, H.V.F.\& Silva, Y.S. (2019). A influência da prática de ginástica localizada na qualidade de vida das pessoas idosas. Gep News, 2 (2), 82-89.

United Nations. (2020). World population ageing 2020 highlights: living arrangements of older persons. Department of Economic and Social Affairs, Population Division. 2020_world_population_ageing_highlights.pdf. https://www.un.org/development/desa/pd/sites/www.un.org.development.desa.pd/files/undesa_pd- 
Research, Society and Development, v. 10, n. 6, e28010615879, 2021

(CC BY 4.0) | ISSN 2525-3409 | DOI: http://dx.doi.org/10.33448/rsd-v10i6.15879

Vala, J.A. (2003). Análise de Conteúdo. In Silva A. \& Pinto, J. (Orgs.), A Metodologia das Ciências Sociais (101-128). Porto: Edições Afrontamento.

World Health Organization. (2017). Global strategy and action plan on ageing and health. World Health Organization. http://www.who.int/ageing/WHOGSAP-2017.pdf?ua=1.

Yin, R.K. (2016). Pesquisa qualitativa do início ao fim. Porto Alegre, RS: Penso. 